Recent Advances in Communication, Electronics \& Electrical Engineering
गन्धालया:
INTERNATIONAL JOURNAL OF RESEARCH -
GRANTHAALAYAH
A knowledge Repository

RACEEE -

17

\title{
DESIGN AND DEVELOPMENT OF LOW COST SMART BILLING SYSTEM
}

\author{
Prakruthi K ${ }^{1}$, Sowmya B ${ }^{2}$, Kiran $B^{3}$, Manjunatha K N ${ }^{4}$ \\ ${ }^{1,2}$ Department of Information Science Engineering, School of Engineering and Technology, Jain \\ University, India \\ ${ }^{3,4}$ Department of Electronics and Communication Engineering, School of Engineering and \\ Technology, Jain University, India
}

DOI: https://doi.org/10.29121/granthaalayah.v5.i4RACEEE.2017.3336

\begin{abstract}
This paper presents the design of smart billing systems which can be placed to reduce the huge crowd in the malls in towns and cities. Especially it becomes more crowded on holidays. Customers pick different items in the malls and put them into trolley. At the cash counter billing process is done using bar code scanner and it is very time consuming process. To avoid this we are developing a system which we called as "Smart Billing System Using RFID and ZIGBEE". In this system we are using RFID tags instead of barcodes. This RFID tag will be on the products. Whenever the customer puts a product into the trolley it will get scanned by RFID reader and product name and cost will be displayed on visual display. Like this the process goes on. We are using ZIGBEE transceiver which will be on the trolley which is used to transfer data to main computer. At the main computer ZIGBEE transceiver will be placed which will receive data from transmitter. To store the products price and total billing controller memory is used. Product name and the total cost will be displayed on LCD.
\end{abstract}

Keywords: LPC 2148; RFID Tag; ZIGBEE.

Cite This Article: Prakruthi K, Sowmya B, Kiran B, and Manjunatha K N. (2017). "DESIGN AND DEVELOPMENT OF LOW COST SMART BILLING SYSTEM." International Journal of Research - Granthaalayah, 5(4) RACEEE, 141-146.

\section{Introduction}

Shopping mall is a place where people get their daily necessities ranging from food products, clothing, electronic appliances etc. Now a day's number of shopping malls has increased throughout the global due to public demand and spending. 
Recent Advances in Communication, Electronics \& Electrical Engineering

The basic idea of this is used in the Malls and Shopping Centers. Barcodes have been in existence for many years and have been used by departmental stores and supermarkets to manage purchases of merchandize by customers and keep track of inventory. However, the barcode system is no longer the best way to business operation. Customers are tired of waiting in long, slowly moving checkout line in departmental stores, especially, in holidays.

Here is a system with these three main benefits: It creates a better shopping experience for the customers by saving their time. It minimizes the man power required at shopping mall, as the checking out process at the check-out counters is eliminated altogether. Finally It handles the cases of deception if any, thereby making the system attractive not only to the customers but also the sellers.

With the decrease of prices through efficiencies of technology and large-scale production of semiconductor wireless components, there has been a search for new markets in which semiconductor chips can be used. This has led to the use of RFID also known as smart tags. RFID stands for Radio Frequency Identification.

\section{Methodology}

\subsection{Materials}

RFID TAGS: An RFID tag [1] is comprised of a microchip containing identifying information and an antenna that transmits this data wirelessly to a reader. At its most basic, the chip will contain a serialized identifier, or license plate number, that uniquely identifies that item, similar to the way many bar codes are used today. A key difference, however is that RFID tags have a higher data capacity than their bar code counterparts. This increases the options for the type of information that can be encoded on the tag, including the manufacturer, batch or lot number, weight, ownership, destination and history (such as the temperature range to which an item has been exposed). In fact, an unlimited list of other types of information can be stored on RFID tags, depending on application needs. An RFID tag can be placed on individual items, cases or pallets for identification purposes, as well as on fixed assets such as trailers, containers, totes, etc. RFID Reader RFID Proximity OEM Reader Module has a built-in antenna in minimized form factor.

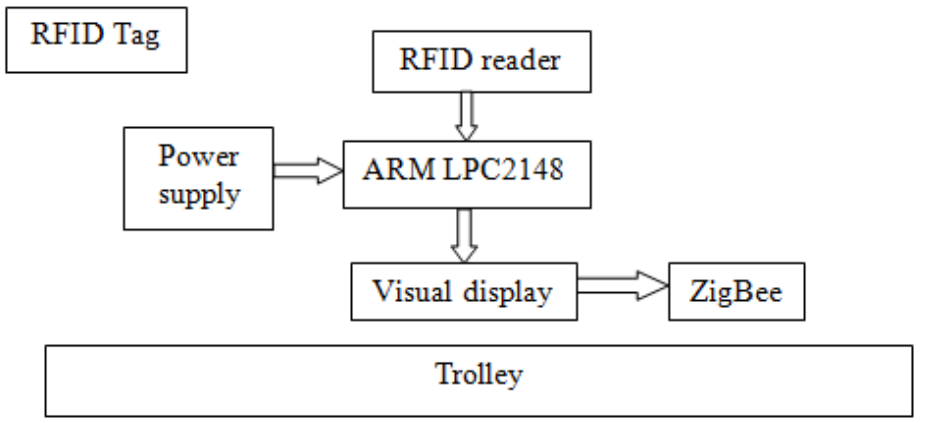

Figure 1: Block diagram of system at trolley - TX section 
Recent Advances in Communication, Electronics \& Electrical Engineering

It is designed to work on the industry standard carrier frequency of $125 \mathrm{kHz}$. This LF reader module with an internal or an external antenna facilitates communication with Read-Only transponders - type UNIQUE or TK5530 via the air interface. The tag data is sent to the host systems via the wired communication interface with a protocol selected from the module Both TTL and Wiegend Protocol.

LPC2148 Microcontroller: The LPC2148 microcontrollers [5] are based on a 32/16 bit ARM7TDMI-S CPU with real-time emulation and embedded trace support, that combines the microcontroller with embedded high speed flash memory ranging from $32 \mathrm{~Kb}$ to $512 \mathrm{~Kb}$. A 128 bit wide memory interface and unique accelerator architecture enable 32-bit code execution at the maximum clock rate. For critical code size applications, the alternative 16-bit Thumb mode reduces code by more than $30 \%$ with minimal performance penalty. Due to their tiny size and low power consumption, LPC2148 are ideal for applications where miniaturization is a key requirement, such as access control and point-of-sale. A blend of serial communications interfaces ranging from a USB 2.0 Full Speed device [2], multiple UARTS, SPI, SSP to I ${ }^{2} C^{\prime}$ 's and on-chip SRAM of $8 \mathrm{~Kb}$ up to $40 \mathrm{~Kb}$, make these devices very well suited for communication gateways and protocol converters, soft modems, voice recognition and low end imaging, providing both large buffer size and high processing power. Various 32-bit timers, single or dual 10-bit ADC(s), 10-bit DAC, PWM channels and 45 fast GPIO lines with up to nine edge or level sensitive external interrupt pins make these microcontrollers particularly suitable for industrial control and medical systems.

16 X 2 LCD Liquid Crystal Display (LCD): A LCD is a thin, flat panel used for electronically displaying information such as text, images, and moving pictures. Its uses include monitors for computers, televisions, instrument panels, and other devices ranging from aircraft cockpit displays, to every-day consumer devices such as video players, gaming devices, clocks, watches, calculators, and telephones. Among its major features are its lightweight construction, its portability, and its ability to be produced in much larger screen sizes than are practical for the construction of cathode ray tube (CRT) display technology. Its low electrical power consumption enables it to be used in battery-powered electronic equipment. It is an electronically-modulated optical device made up of any number of pixels filled with liquid crystals and arrayed in front of a light source (backlight) or reflector to produce images in color or monochrome.

ZigBee is the most popular industry wireless mesh networking standard for connecting sensors, instrumentation and control systems. ZigBee is a specification for communication in a wireless personal area network (WPAN). ZigBee and IEEE 802.15.4 [4] are low data rate wireless networking standards that can eliminate the costly and damage prone wiring in industrial control applications. 
Recent Advances in Communication, Electronics \& Electrical Engineering

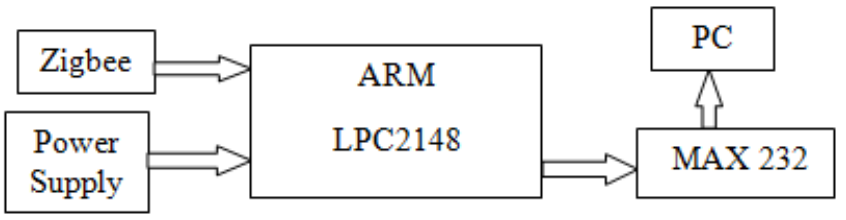

Figure 2: Block diagram of system at trolley - RX section

MAX232 Interface: The MAX232 is a dual driver/receiver that includes a capacitive voltage generator to supply EIA-232 voltage levels from a single 5-V supply. Each receiver converts EIA-232 inputs to 5-V TTL/CMOS levels. These receivers have a typical threshold of $1.3 \mathrm{~V}$ and a typical hysteresis of $0.5 \mathrm{~V}$, and can accept $\pm 30-\mathrm{V}$ inputs. Each driver converts TTL/CMOS input levels into EIA-232 levels.

PC [Main Computer]: After all the shopping of the person, the total cost and products in the trolley will be sent to the main server with the help of ZIGBEE. The ZIGBEE transmitter will send the information from microcontroller and ZIGBEE receiver will collect the data and sends it the main computer at the billing counter. In this module, we are transferring the information of the products and its total cost to the main computer which will be kept at the billing station.

\section{Results and Discussions}

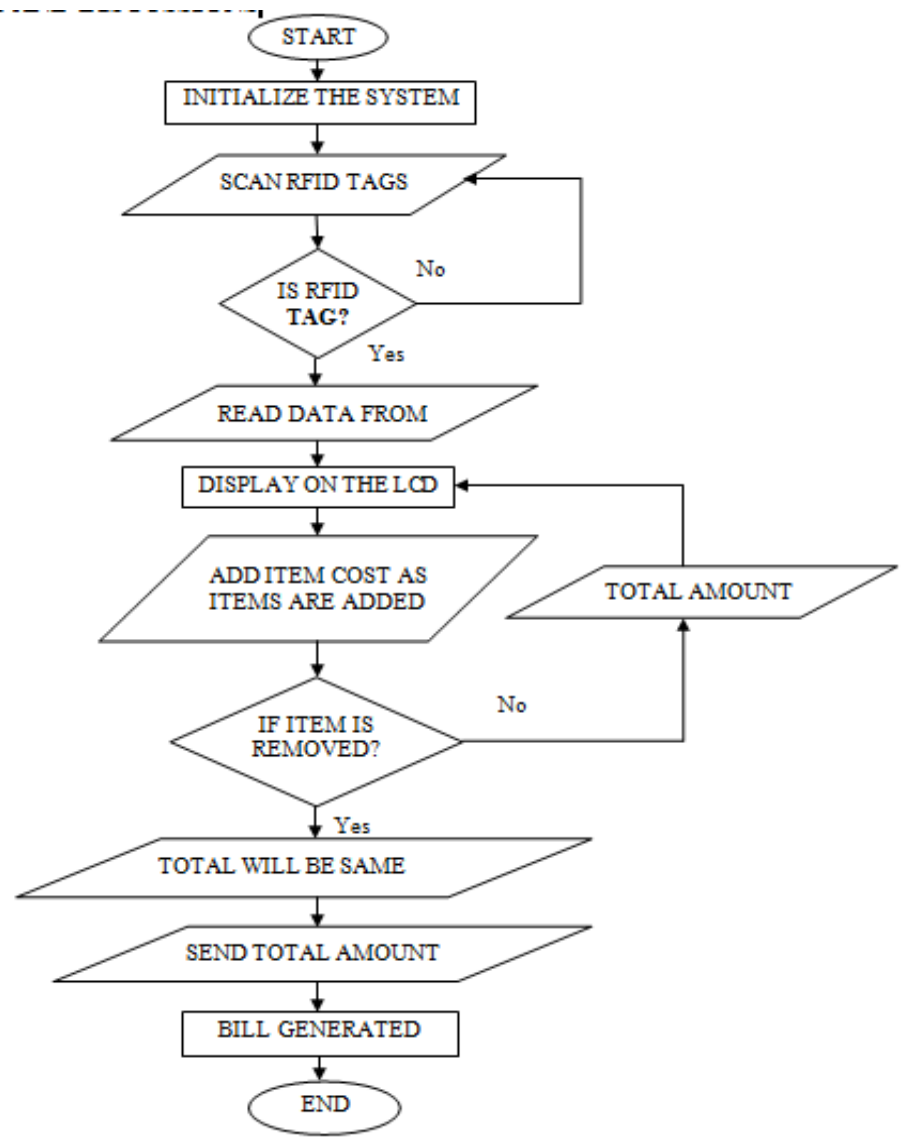

Figure 3: Flow chart of the system 


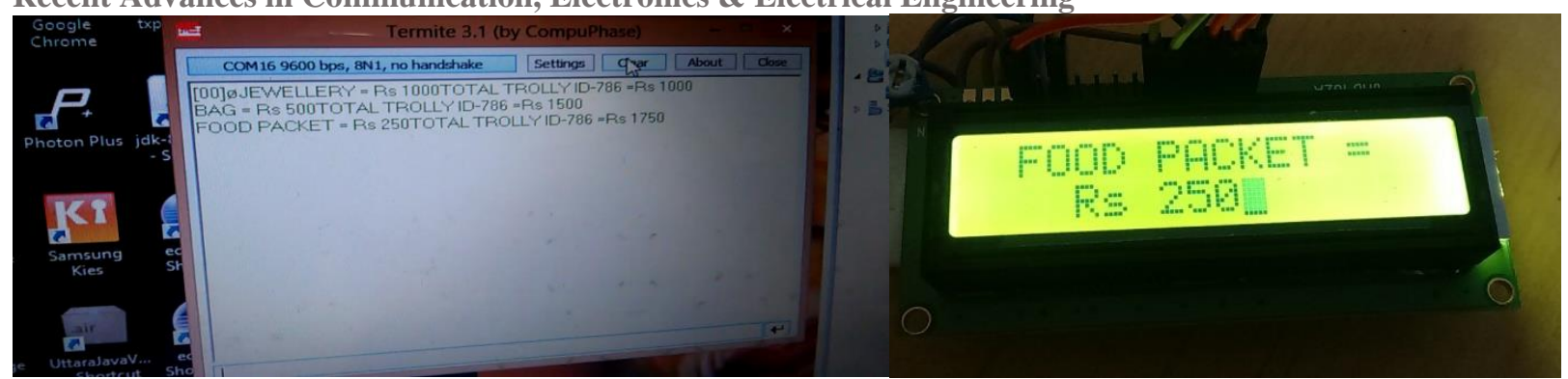

Figure 4: Terminate display

Figure 5: LCD display

All the products in mall are equipped with RFID tag. When a person puts in an item, the reader reads the tag within the range of $5 \mathrm{~cm}$. The code that is read by the reader is then sent to the controller which displays in LCD that is interfaced to LPC2148. The LCD interfaced to controller displays the trolley number, product name and price.

As we put the items, the costs will get added to total. Thus billing is done in the trolley. If we want to remove some inserted item then we toggle the switch to decrement the products. Thus items cost gets subtracted and is displayed on LCD. At billing counter the total bill data will be transferred to PC by the wireless ZigBee transmitter interfaced with processor. It is $2.4 \mathrm{GHz} \mathrm{RF}$ module which works in free ISM band.

In this model, we are transferring the information of the products and its total cost to the main computer which will be kept at the billing station. The information is sent through Zigbee via serial communication. The information is displayed on PC Termite graphical user interface which is configured to RS232 terminal. It uses an interface similar to that of messenger or chat programs, with a large window for all received data and an edit line for typing in strings to transmit.

Table 1: Observed results

\begin{tabular}{|l|l|l|}
\hline \multicolumn{1}{|c|}{ MODEL } & \multicolumn{1}{|c|}{ RANGE } & RESULT \\
\hline RFID & $1 \mathrm{~cm}$ & Good \\
\cline { 2 - 3 } \multirow{2}{*}{$\begin{array}{l}\text { Reader(125khz/LF) } \\
\text { RS232 TTL O/P }\end{array}$} & $2 \mathrm{~cm}$ & Good \\
\cline { 2 - 3 } & $3 \mathrm{~cm}$ & Good \\
\cline { 2 - 3 } & $4 \mathrm{~cm}$ & Good \\
\cline { 2 - 3 } & $5 \mathrm{~cm}$ & Good \\
\cline { 2 - 3 } & $5.5 \mathrm{~cm}$ & Fair \\
\cline { 2 - 3 } & $6 \mathrm{~cm}$ & Fair \\
\cline { 2 - 3 } & $7 \mathrm{~cm}$ & Not Detected \\
\hline ZIGBEE & $85 \mathrm{~m}$ & Good \\
\cline { 2 - 3 } & $90 \mathrm{~m}$ & Good \\
\cline { 2 - 3 } & $95 \mathrm{~m}$ & Good \\
\cline { 2 - 3 } & $100 \mathrm{~m}$ & Good \\
\cline { 2 - 3 } & $105 \mathrm{~m}$ & Fair \\
\hline
\end{tabular}




\section{Conclusions \& Recommendations}

This work highlights that, idea of this after observing the long queues at the sales and billing counters at the shopping malls and retail bazaars. The intended objectives were successfully achieved in the prototype model. The developed product is easy to use, low-cost and does not require any special training.

The architecture of the system can be used in the shopping center for intelligent and easy shopping in the malls to save time, energy and money of the consumers. This system would help in cost saving at the supply chain level. At the same time it would also reduce the required no of salesmen.

\section{Acknowledgements}

The Authors would like thank the management of Jain Group of institutions. The work has been carried out at project laboratory department of Electronics and Communication Engineering, School of Engineering and Technology, Jain University

\section{References}

[1] J.Suryaprasad, B.O.P. Kumar, D. Roopa and A.K. Arjun, "A Novel Low-Cost Intelligent Shopping Cart", IEEE 2nd International Conference on Networked Embedded Systems for Enterprise Applications, pp.1-4, 2011.

[2] D.V.S Chandra Babu, "wireless intelligent billing trolley for supermarket", International Journal of Advanced Research in Technology, vol.3, issue 1, Aug. 2012.

[3] Mr.P. Chandrasekar., "Smart Shopping Cart with Automatic Billing System through RFID and ZigBee"

[4] Roussos G, BirkbeckColl. "Enabling RFID in retail”, IEEE Computer Magazine, Vol.39, Issue: 3PP: 25 - 30, 20 March 2006.

[5] Floerkemeier C.,Sarma S., "An overview of RFID System Interfaces and Reader Protocol”, 2008 IEEE International Conference.

[6] Thorsten Blecker and George Haung "RFID in Operations and Supply Chain Management" 2008.

\footnotetext{
*Corresponding author.

E-mail address: prakruthi713@gmail.com
} 